ÉGYPTE monde arabe

\section{Égypte/Monde arabe}

15-16 | 1993

Les crises soudanaises des années 80

\title{
Les réfugiés étrangers au Soudan
}

\section{Salah al-Din al-Shazali}

\section{(2) OpenEdition}

\section{Journals}

Édition électronique

URL : https://journals.openedition.org/ema/1095

DOl : 10.4000/ema.1095

ISSN : 2090-7273

\section{Éditeur}

CEDEJ - Centre d'études et de documentation économiques juridiques et sociales

\section{Édition imprimée}

Date de publication : 31 décembre 1993

Pagination : 175-190

ISSN : 1110-5097

\section{Référence électronique}

Salah al-Din al-Shazali, « Les réfugiés étrangers au Soudan », Égypte/Monde arabe [En ligne], 15-16। 1993, mis en ligne le 08 juillet 2008, consulté le 07 juillet 2022. URL : http://journals.openedition.org/ ema/1095 ; DOI : https://doi.org/10.4000/ema.1095

Ce document a été généré automatiquement le 7 juillet 2022.

Tous droits réservés 


\title{
Les réfugiés étrangers au Soudan
}

\author{
Salah al-Din al-Shazali
}

\section{NOTE DE L'ÉDITEUR}

Traduit de l'anglais par Samia Rizq.

1 Au cours des trois dernières décennies, le Soudan a accueilli un nombre croissant de réfugiés venus surtout du Tchad, de l'Ethiopie, de l'Ouganda et du Zaïre. Conflits armés, guerres civiles et désastres naturels ont poussé bon nombre de ressortissants de ces pays à franchir la frontière. Bien qu'il soit difficile d'en connaitre le nombre exact eh raison de leur constante mobilité - à l'intérieur du Soudan comme en direction ou en provenance de ce pays - certaines estimations prudentes, confirmées par le HCR (Haut comité de l'ONU pour les réfugiés), indiquent néanmoins des chiffres représentant pour le pays un lourd fardeau : en 1984-85, au moment où la famine atteignait son apogée, on comptait en effet plus d'un million de réfugiés, ce qui signifie que le rapport réfugiés/ nationaux était de l'ordre de 1/120 - un des plus élevés du monde sans aucun doute. Si, depuis, la majorité des réfugiés victimes de la sécheresse ont regagné volontairement leur pays d'origine, par contre, la guerre, spécialement en Érythrée et au Tchad, a obligé un nombre toujours croissant de personnes à fuir vers le Soudan.

2 En décembre 1990, les réfugiés représentaient presque un million de personnes; le tableau 1 fournit une vue d'ensemble du nombre et de l'origine des réfugiés à cette époque.

Tableau 1 : Nombre et origine des réfugiés étrangers au Soudan

\begin{tabular}{|l|l|l|}
\hline Pays d'origine & Nombre & $\%$ \\
\hline \hline Zaïre & 5.000 & 1 \\
\hline \hline Ouganda & 5.000 & 1 \\
\hline
\end{tabular}




\begin{tabular}{|l|l|l|}
\hline Tchad & 110.849 & 11 \\
\hline \hline Ethiopie & 838.450 & 87 \\
\hline \hline Total & 959.299 & 100 \\
\hline
\end{tabular}

Source : COR.

3 La répartition des réfugiés sur l'ensemble du territoire soudanais est cependant inégale. Le tableau $\mathrm{n}^{\circ} 2$ montre la tendance nette de ceux-ci à se concentrer dans les régions de l'Est, l'Equatoria, le Darfour, ainsi que dans les banlieues du GrandKhartoum.

Tableau 2 : Répartition des réfugiés étrangers par région et par type de logement

\begin{tabular}{|l|l|l|l|l|}
\hline Région & Camps & Regroupements spontanés & Total & $\%$ \\
\hline \hline Est & 307.686 & 46.500 & 772.686 & 80 \\
\hline \hline Centre & 10.764 & 20.000 & 30.764 & 3 \\
\hline \hline Darfour & 22.849 & 88,000 & 110.849 & 11 \\
\hline \hline Equatoria & - & 5.000 & 5.000 & 1 \\
\hline \hline Khartoum & - & 40.000 & 40.000 & 5 \\
\hline \hline Total & 341.299 & 618.000 & 959.299 & - \\
\hline \hline \% & $35 \%$ & $65 \%$ & $100 \%$ \\
\hline
\end{tabular}

Source : COR.

4 La concentration des réfugiés est nettement plus importante à l'est, où ils représentent $33 \%$ de l'ensemble de la population. Par rapport à sa densité relativement faible et à l'insuffisance de l'infrastructure, la région Est regroupe un ensemble de réfugiés considérable si on la compare à la plupart des régions d'accueil dans le monde. La plupart viennent de la frontière éthiopienne, plus particulièrement de l'Érythrée et du Tigré.

Politique de l'État à l'égard des réfugiés

La stratégie déclarée du gouvernement soudanais consiste à encourager les réfugiés à se prendre en charge. Dans ce but, un programme en trois étapes a été adopté. Durant la première étape, dite d'assistance, les besoins essentiels en matière d'alimentation, de soins médicaux, de logement, etc., sont assurés par l'État. L'objectif de la deuxième étape, dite d'insertion, est de relever le niveau de vie des réfugiés pour qu'il atteigne celui des Soudanais habitant la même région. L'insertion est supposée achevée lorsque les réfugiés sont installés dans des agglomérations rurales ou semi-urbaines. Durant la troisième étape, celle dite du développement, les réfugiés ne reçoivent plus de 
subventions particulières et ne bénéficient que des avantages sociaux dont bénéficient les nationaux dans le cadre du plan de développement.

Les catégories de réfugiés

6 La mise en place de cette stratégie dépend de l'installation des réfugiés dans des camps réservés à cet effet, loin des communautés soudanaises de la région. Cependant, malgré l'aide que le gouvernement soudanais, le HCR et d'autres institutions apportent à ces camps gérés par l'État, des réfugiés choisissent parfois de s'installer ailleurs. Résultat de cette "insoumission", ils sont classés en deux catégories: d'une part, celle des réfugiés « reconnus » comme tels par l'État, qui résident dans les camps à eux destinés, ainsi qu'une minorité (le plus souvent des étudiants) autorisée à se loger en milieu urbain ; d'autre part, les réfugiés ayant pris en charge leur propre installation au sein de la population soudanaise et qui, de ce fait, n'ont pas le statut légal de réfugiés.

Les camps de réfugiés

7 Signataire des conventions de Genève et de l'OUA sur le statut des réfugiés, le gouvernement soudanais s'est engagé à assurer à ces derniers des installations situées à 50 mètres au moins des frontières de leur pays d'origine. En même temps, il entend considérer comme transitoire le séjour des réfugiés et s'attend à ce qu'ils choisissent le rapatriement volontaire dès l'amélioration de la situation de leur pays. Le gouvernement soudanais tente par ailleurs de limiter le fardeau que représente cette immigration massive pour l'économie et la société soudanaises. Dans la mesure où l'économie nationale a déjà des difficultés à répondre aux besoins élémentaires d'une grande partie de la population, le gouvernement demande, au titre d'aide aux réfugiés, le soutien de la communauté internationale, principalement représentée par le HCR. C'est pour cette raison et pour d'autres - relatives à la sécurité - que les autorités soudanaises ont cherché à regrouper les réfugiés dans des camps - organised settlements - qui leur sont réservés. Le HCR et les diverses organisations concernées par l'aide aux réfugiés préconisent d'ailleurs ce type d'installations, officiellement appelées "zones d'hébergement des réfugiés ", gérées par l'État et isolées.

Les camps de réfugiés ont d'abord été construits en 1969 lorsque le gouvernement, en accord avec le HCR, a décidé de transférer la première vague de réfugiés érythréens à Gala'a al-Nahal dans la province de Kassala (est du pays). Dans ces land settlements, les formes traditionnelles d'agriculture et d'élevage suffisaient aux besoins de la communauté. L'infrastructure (routes, eau, services de santé et d'éducation, etc.) était assurée grâce à une aide financière extérieure (fournie par l'intermédiaire du HCR) destinée à profiter aussi bien aux réfugiés qu'aux communautés soudanaises environnantes.

9 Les Iand settlements sont situés à la périphérie, dans des zones faiblement peuplées, voire désertes. Outre la parcelle de terrain qui leur est allouée, les réfugiés reçoivent, pour quelques années, une indemnité et une aide alimentaire jusqu'à ce qu'ils puissent se prendre en charge. Les habitants de ces settlements parviennent parfois à acquérir du bétail. Ces agglomérations doivent toutefois se contenter d'une quantité d'eau et d'une surface de terrain limitées.

10 Avec l'arrivée d'un nombre croissant de réfugiés, le gouvernement soudanais a fini par reconsidérer la question de l'allocation de terrains. Loin d'être des terrains vagues comme on l'avait cru, les parcelles affectées aux communautés de réfugiés étaient des pâturages utilisés par des pasteurs nomades de manière saisonnière. Devant l'amertume suscitée par l'allocation de terres agricoles (et la reconnaissance implicite, 
de la part du gouvernement, du risque de l'installation à long terme des bénéficiaires), deux autres types d'hébergement pour réfugiés ont été mis en place : les camps pour agriculteurs salariés (wage-earning rural settlements) et les camps péri-urbains (periurban settlements).

11 Comme leur nom l'indique, les premiers fournissent un travail salarié aux agriculteurs et sont situés aux confins des vastes systèmes d'irrigation de New Halfa et de Rahad, ou à proximité des systèmes d'irrigation mécanisée dans la région du Grand-Gedaref. Les camps péri-urbains établis autour de Gedaref. Port-Soudan et Khashm al-Girba devraient, d'une certaine façon, permettre aux réfugiés de trouver un emploi en zone urbaine; leur organisation tient relativement compte du fait que tous les réfugiés ne sont pas forcément préparés au travail d'agriculteur ou qu'ils n'ont pas tous l'intention de s'y engager. L'installation de réfugiés dans différents types de camps gérés par l'État n'a réussi qu'en partie. Comme l'indique le tableau 2 , seuls $35 \%$ des réfugiés résident réellement dans ces communautés. Les raisons qui poussent certains d'entre eux à les quitter sont variées et complexes. Elles peuvent être liées aux campagnes de mobilisation menées par les fronts de libération pour leurs opérations militaires audelà des frontières : ces derniers considèrent probablement que les camps constituent un obstacle à leurs opérations, les réfugiés y résidant étant le plus souvent dépolitisés et non armés, et la situation qui les a poussés à la fuite (lutte contre le régime du pays d'origine ou. comme dans le cas de l'Érythrée, libération du colonialisme éthiopien) non encore résolue.

Par ailleurs, la gestion étatique des camps semble constituer, par nature, un facteur décourageant : un des problèmes majeurs de ces structures est qu'elles ne possèdent pas d'autosuffisance économique et ne sont pas près d'y parvenir rapidement. Au mieux, dans les camps les plus performants, l'aide alimentaire n'est plus nécessaire et la population peut relativement subvenir à ses besoins. Cette apparente viabilité pourrait cependant être minée si l'aide fournie aux réfugiés sous forme de services gratuits ou largement subventionnés (santé, éducation, denrées agricoles) venait à être interrompue. C'est ce qui s'est produit dans les camps de regroupement d'Érythréens de la région de Gala'a al-Nahal. Même ce succès limité n'a pas été facile à réaliser. Un des facteurs qui ont contribué à rendre ces premières installations invivables a été le faible potentiel de la région où elles étaient implantées.

Les agriculteurs salariés ont à pâtir d'une situation doublement difficile : les salaires bas et le travail saisonnier. On leur demande de se prendre en charge alors que la maind'œuvre agricole au Soudan, comme on le sait, ne peut vivre du seul salaire d'un emploi saisonnier mais compte aussi sur ses traditionnelles pratiques d'économie paysanne pour subvenir à ses besoins pendant le reste de l'année. Or, contrairement aux agriculteurs soudanais, les réfugiés n'ont pas accès aux activités traditionnelles liées à l'économie paysanne.

Il peut sembler paradoxal qu'un des camps d'agriculteurs salariés les plus performants n'ait pas été, au départ, établi à cet effet: le centre d'accueil de Wad Sharifie, à proximité de la ville de Kassala, a été démantelé de crainte de le voir se transformer en camp permanent. En plus de l'aide alimentaire et des autres services dont ils bénéficiaient, les réfugiés de ce centre avaient accès à des emplois dans l'horticulture dans le cadre de projets d'irrigation naturelle mécanisée récemment mis en place, mais non planifiés, dans la région de Gargaf (district sud de Kassala) aussi bien que dans la ville de Kassala. Toutefois, le centre d'accueil étant situé à moins de 50 miles des 
frontières, le COR et le HCR avaient hâte de le fermer et de transférer les réfugiés dans des camps "moins viables" mais plus éloignés des frontières. A cause de ces problèmes, un grand nombre de réfugiés (surtout les plus jeunes, les plus qualifies et ceux provenant de milieux urbains) ont préféré ne pas s'installer dans ces camps. En même temps, ces camps ont attiré un nombre important de personnes incapables d'être autonomes (handicapés, personnes âgées) ou ayant trop de personnes à charge et qui, autrement, auraient eu du mal à subvenir à leurs besoins.

L'installation «spontanée »

15 La majorité des réfugiés ont donc choisi l'immersion dans les communautés locales. Mais en agissant de la sorte, ils se sont privés d'une reconnaissance légale de leur statut. L'ambiguïté de leur situation provoque en eux un sentiment d'insécurité et, de plus, ils n'ont pas droit à l'aide régulière dont bénéficient ceux qui, résidant dans les camps gérés par l'État, ont le statut légal de réfugiés.

De manière générale, les réfugiés "spontanés " des zones rurales sont analphabètes, anciens fermiers et pasteurs ayant longtemps appartenu à des groupes vivant près des frontières et qui, par conséquent, parviennent assez facilement à rejoindre les tribus frontalières de la région. Les premiers arrivés ont pu acquérir des droits d'usufruit sur des terres agricoles conformes aux normes traditionnelles en vigueur. Cependant, depuis quelques années, ils ont davantage de difficultés à obtenir de tels privilèges. Ils tentent alors de louer des terres, d'entrer dans des copropriétés ou de se faire engager comme travailleurs occasionnels dans l'agriculture, l'élevage, comme bûcherons ou dans l'industrie du charbon de bois. Le fait de se trouver dans des zones frontalières facilite leur déplacement en provenance et en direction de leur pays d'origine. Certains ont réussi non seulement à rester en contact avec la famille, de l'autre côté de la frontière, mais aussi à y exercer des activités lucratives. Dans les régions de l'est, les réfugiés érythréens, qui parfois n'ont pas droit à des terres, continuent à avoir des fermes traditionnelles au-delà des frontières. D'autres travaillent comme bûcherons dans les forêts encore riches de l'Érythrée et transportent, à dos d'âne, le bois vers le Soudan. En outre, les pasteurs préfèrent être à proximité des frontières de manière à emmener leurs troupeaux dans des pâturages relativement riches mais abandonnés. Cela suppose cependant qu'ils versent des pots-de-vin aux douaniers.

Les installations spontanées des villes sont plus complexes. Au départ, elles étaient réservées aux réfugiés venant de zones urbaines, c'est-à-dire aux .personnes exerçant des fonctions telles que fonctionnaires, enseignants ou employés ayant reçu une formation professionnelle dans le secteur des services. Sont également inclus dans cette catégorie les étudiants inscrits dans des établissements d'enseignement. Toutes ces catégories ne peuvent pas vivre en zone rurale; d'une part, elles n'ont pas la compétence requise pour travailler dans l'agriculture ou l'élevage; d'autre part, dès le début, elles n'ont eu que peu ou pas de difficultés à trouver un emploi en zone urbaine. Quant aux réfugiés titulaires de diplômes supérieurs, ils sont rarement intéressés par la perspective de travailler et de s'installer au Soudan, et aspirent à gagner l'Europe ou les États-Unis. Tel est leur rêve, voire l'objectif qu'ils attendent patiemment de pouvoir réaliser.

L'origine et les caractéristiques sociales des réfugiés spontanés des zones urbaines ont changé au cours de ces dernières années. Un nombre croissant de personnes qui, dans leur pays d'origine, vivaient en zone rurale, viennent s'installer dans les grandes villes, 
le plus souvent comme squatters, nouveau noyau de travailleurs non qualifiés, vivant d'emplois occasionnels ou clandestins.

Soulignons que la migration rurale-urbaine des réfugiés spontanés ne devrait pas être interprétée uniquement selon une perspective étroite centrée sur les conditions spécifiques des camps gérés par l'État. Le niveau de vie dans les campagnes soudanaises est généralement bas, et l'exode rural des Soudanais au moins aussi important que la migration rurale-urbaine des réfugiés.

L'impression générale est qu'il n'y a quasiment aucune différence entre les conditions de vie des réfugiés vivant dans les camps gérés par l'État et subventionnés, et celles de la majorité pauvre des réfugiés spontanés. Se pose alors la question de savoir où passe le budget consacré à l'aide aux réfugiés. Malgré les sommes dépensées pour les camps, rien ne permet de dire que ceux qui ont le statut de réfugiés sont plus favorisés que les autres. Ce qui signifie, ou bien que l'aide accordée ne suffit pas à couvrir les besoins réels de ces camps, ou bien que la nature de cette aide ne répond pas véritablement aux besoins des réfugiés.

Impact des réfugiés sur la région d'accueil

21 L'impact des réfugiés sur la société, l'économie et l'environnement soudanais, surtout dans les régions où leur concentration est relativement forte, est sujet à controverse tant chez les chercheurs que chez les responsables gouvernementaux et le personnel des organisations internationales. Les débats portent principalement sur la question de savoir si l'influence exercée par les réfugiés est positive ou négative et, en termes monétaires, quelle « valeur » il faudrait lui attribuer. Toutefois, la question primordiale concernant la méthodologie à adopter pour déterminer la nature exacte de l'impact luimême n'est pas résolue.

Evaluer de manière précise l'impact des réfugiés est une tâche difficile, voire impossible. L'affluence des réfugiés n'est qu'un des multiples facteurs de changement (démographique, social, politique, sécuritaire, climatologique, etc.) qui, par leur interaction, exercent une influence continue, quoique de nature et d'intensité variables, sur la vie socio-économique des régions d'accueil (Kuhlman et al., 1987). Cette affluence, à proprement parler, n'est qu'une des composantes de la croissance démographique. Les autres composantes sont constituées par la croissance naturelle de la population dans chaque région et les différentes formes de migration interne. Ainsi, lorsque l'affluence de réfugiés étrangers a atteint son apogée en 1984-85, la région Est du pays a vu arriver en même temps les victimes de la sécheresse qui sévissait à l'ouest et au sud du Soudan. Ce n'est que sur la base d'une évaluation globale des processus de changement en cours dans les différentes régions que l'on peut raisonnablement tenter de s'interroger sur la part respective de chacun de ces facteurs. C'est la méthodologie que nous avons adoptée ici pour étudier l'impact des réfugiés.

Par ailleurs, cette étude ne prétend pas déterminer, à travers une évaluation qualitative, si l'impact a été positif ou négatif. Car un même facteur (hausse des loyers, par exemple) peut être positif pour les uns (propriétaires) et négatif pour les autres (locataires). Cet article se propose donc d'étudier les effets de l'affluence des réfugiés sur :

- le niveau et l'efficience des services publics ;

- les ressources naturelles ;

- le marché de l'emploi et les processus de production ;

- La cherté de la vie pour différentes catégories sociales. 
Impact sur les services sociaux et l'infrastructure 
résoudre un problème qui ne peut être attribué aux seuls réfugiés. A titre d'exemple, le HCR envoie de temps à autre des containers de médicaments dans l'est du pays. Or, comme cela se produit souvent, les autorités locales ont mal à se procurer ces médicaments pour la population soudanaise elle-même. Cependant, ce type d'assistance n'est pas régulier et ne permet d'assurer ni les besoins urgents ni les besoins d'équipement (laboratoires, services de chirurgie, lits, draps, matériel chirurgical, etc.) Dans le domaine de l'éducation, le HCR subventionne uniquement l'enseignement primaire : le coût de l'éducation des réfugiés aux niveaux intermédiaire et secondaire est donc à la charge du gouvernement et/ou de la population.

Dans le domaine des infrastructures, les services tels que l'eau et l'électricité sont bien loin du niveau souhaité par le gouvernement et par la population (réfugiés et Soudanais). Le mode de fonctionnement des agences des Nations Unies ne permet guère au Soudan d'en bénéficier. Le HCR, qui n'a qu'un mandat d'assistance à court terme, ne s'aventure pas dans des projets de "développement " qui sont du ressort d'autres organisations des Nations Unies (FAO, OMS, etc.) A leur tour, les organisations spécialisées répondent à la demande de chaque pays mais ne fournissent pas, en principe, d'aide supplémentaire pour compenser la présence de réfugiés, et soutiennent que ces 'derniers relèvent du HCR. Les missions regroupant des représentants de plusieurs organisations devraient résoudre ce problème. Mais quoique plusieurs d'entre elles soient déjà venues au Soudan, ce pays attend toujours que soit approuvé le financement de projets dans les régions d'accueil des réfugiés.

Impact sur les ressources naturelles

31 La plus grande partie du Soudan connaît une dégradation intense du milieu naturel, due pour partie à la sécheresse qu'ont connue les dernières décennies et pour partie à l'action humaine. On sait que le forage de puits contribue à la désertification et au déboisement dans leur périphérie. La coupe du bois devant servir de combustible et le surpâturage résultant de la marginalisation des pasteurs nomades par l'implantation, sur les terrains traditionnellement réservés aux pâturages, de projets agricoles à grande échelle; y contribuent également. On ne peut donc considérer les réfugiés comme seuls responsables de la détérioration du milieu naturel dans ces régions, mais il ne faut pas non plus sous-estimer leur impact.

Tout d'abord, l'installation des camps de réfugiés a contribué de manière considérable au processus de déboisement massif. Dans la province de Kassala, le gouvernement a défriché une superficie totale de 126.000 feddans pour installer les réfugiés sur des terres agricoles. Par ailleurs, dans la mesure où la majorité des réfugiés des zones rurales séjournent dans des implantations spontanées, on doit présumer que d'autres superficies, non déterminées, ont également été déboisées. Autre facteur d'aggravation du déboisement $:$ l'utilisation, par les réfugiés comme par les Soudanais, du bois comme combustible.

33 La main-d'œuvre relativement bon marché que constituent les réfugiés a enfin accéléré le déboisement de manière indirecte, les entrepreneurs les ayant engagés comme bûcherons pour des opérations à grande échelle. De plus, les forêts de l'Est continuent d'être le principal fournisseur de combustible et de charbon de bois pour toutes les grandes villes de la région et jusqu'au Grand-Khartoum.

34 Les réfugiés exercent souvent des activités pastorales et possèdent des troupeaux de taille variable. Selon une estimation de 1988, les réfugiés des camps gérés par l'État dans la seule région de Kassala détiennent environ 60.000 têtes de bétail (al-Shazali, 
1988); En outré, les réfugiés contribuent à l'accroissement des ressources animales car ils approvisionnent les marchés régionaux en viande : ils importent la viande appelée karur, que l'on trouve dans les régions de Gedaref et de Kassala, en ramenant du bétail d'Érythrée pour le revendre sur le marché local de la région Est.

La communauté internationale contribue, il est vrai, à la restauration du milieu naturel dans la région (ex. le projet de reboisement des réfugiés de l'Est, projet de coopération monté par Care-Sudan, le Département des forêts et le COR). Cependant, là encore, les efforts déployés sont sans commune mesure avec l'étendue et la gravité de la dégradation environnementale.

Impact sur le marché de l'emploi et les processus de production

L'affluence des réfugiés fournit une main-d'œuvre supplémentaire dans les régions où ils sont établis. Là encore, l'impact considérable de ce phénomène, surtout dans le domaine de l'agriculture, varie en fonction de la situation socio-économique de chaque région : l'augmentation de la main-d'œuvre sera perçue positivement 'ou négativement selon le contexte, comme nous allons le voir.

Lés réfugiés occupent une place de plus en plus importante sur le marché du travail agricole : ils sont notamment engagés dans les projets d'agriculture pluviale mécanisée du Grand-Gedaref et de Gargaf (sud de Kassala). Beaucoup assurent des emplois saisonniers, surtout au moment des récoltes, dans le projet du Gash. Par ailleurs, les réfugiés installés à Girba (projet New Halfa) et à Fau (projet Rahad) constituent une source de main-d'œuvre dont l'importance n'est plus à démontrer.

Dans la région de Kassala, où le taux de réfugiés est le plus important, l'impact de leur présence est particulièrement sensible dans le sous-secteur de l'horticulture, actuellement le plus dynamique de l'économie de la région. L'arrivée des réfugiés ayant constitué un apport important de main-d'œuvre bon marché, la production de fruits et légumes a augmenté de plus de $1.200 \%$ depuis le début des années 70 (Kuhlman et al., op. cit.).

Bien que la majorité des réfugiés vivent en zone rurale, c'est leur présence en zone urbaine qui semble poser le plus de problèmes aux autorités. Cela peut être dû au fait que l'influence des réfugiés sur l'emploi rural correspond largement à la stratégie du gouvernement. Ainsi, dans le cas des camps gérés par l'État, l'impact de leur présence est en grande partie fonction de la politique étatique. Dans certains camps, celle-ci consiste à fournir des emplois salariés aux réfugiés.

Pour ce qui est de la situation du marché de l'emploi en zone urbaine, les inquiétudes semblent fondées sur un certain nombre de considérations ou de présomptions. Si, comme on le pense, le marché de l'emploi n'est pas en mesure d'absorber, pour une raison ou pour une autre, un aussi grand nombre de réfugiés, une catégorie de chômeurs ayant recours à des moyens de survie illégaux devrait émerger. Si par contre, les réfugiés sont absorbés par le marché, le problème qui devrait alors se poser est celui de la concurrence entre main-d'œuvre nationale et main-d'œuvre immigrée.

41 Les réfugiés des zones urbaines se retrouvent dans presque tous les secteurs du marché de l'emploi, mais plus particulièrement dans les transports (conducteurs, mécaniciens, surtout de poids lourds), l'hôtellerie et la restauration, et comme personnel domestique chez des particuliers. Ils ont néanmoins réussi à s'introduire dans d'autres secteurs tels que la construction, les petites entreprises de manufacture et de réparation (ateliers de confection, mécanique, briqueteries, etc.), et dans le commerce (particulièrement de 
marchandises rapportées d'Érythrée). Les seuls emplois auxquels ils n'ont pas accès sont les postes de fonctionnaires (à l'exception du COR). Dans tous les cas, les réfugiés sont présentés soit comme des manœuvres temporaires et inexpérimentés, soit comme une catégorie de pauvres travaillant à leur compte. Une petite minorité arrive à trouver un poste de cadre et rares sont ceux qui travaillent comme employés. Cependant, dans les zones urbaines, un grand nombre de réfugiés sont sans emploi. L'importance du nombre de réfugiés embauchés comme manœuvres temporaires a par ailleurs suscité l'hostilité des travailleurs soudanais et de leurs syndicats professionnels. Les réfugiés sont en effet considérés comme l'un des principaux facteurs de la baisse du niveau de vie que subit la main-d'œuvre urbaine depuis quelques années. Les employeurs semblent toutefois préférer engager des réfugiés car, s'ils ne sont pas nécessairement moins payés que les nationaux, ils font généralement preuve, faute de permis de travail, de davantage de soumission et n'ont pas le droit d'adhérer aux syndicats soudanais.

Dernier point relatif à l'impact des réfugiés sur le marché de l'emploi : on affirme souvent que ces derniers tendent à transgresser la loi du pays d'accueil en s'engageant dans diverses activités clandestines, de la création illégale d'entreprise aux opérations de contrebande. Justifiées en ce qui concerne une partie des réfugiés dans t'Est et dans la région du Darfour, ces accusations doivent toutefois être nuancées compte tenu des facteurs sous-jacents à l'exercice d'activités illicites. A ce propos, il faut insister sur le caractère inconsistant de l'application des lois concernant l'emploi des réfugiés. Alors que la «Régulation of Asylum Act» de 1974 prévoit le droit des réfugiés à l'emploi, ces derniers se voient obligés, dans la pratique, de demander au Labour Office un permis de travail particulier, généralement difficile à obtenir. De plus, ce que l'on entend généralement par emploi, c'est le salariat ; les réfugiés ont beaucoup de mal à obtenir les autorisations nécessaires pour monter leur propre entreprise. Dans une telle conjoncture, ils n'ont que trois possibilités: monter une entreprise clandestine, obtenir une licence sous le nom d'un Soudanais engagé à cet effet ou... demander la citoyenneté soudanaise. Les réfugiés ont recours aux trois stratégies. Si les autorités soudanaises leur avaient facilité l'obtention de permis, ils n'auraient pas eu à transgresser la loi.

Impact sur le niveau de vie

Dans un contexte de crise économique croissante, le niveau de vie a subi une constante dégradation, tout au long de cette décennie, dans toutes les catégories professionnelles. L'arrivée des réfugiés a, sans aucun doute, accentué cette crise en entraînant une augmentation de la demande de produits de base, alors que l'État n'est pas en mesure de satisfaire les besoins nationaux. Pour répondre à cette demande supplémentaire, il doit soit augmenter les importations, ce qui est impossible dans le contexte actuel, soit réduire la quote-part de ses citoyens. Dans la mesure où la plus grande partie du budget provient des impôts indirects sur les biens et les services, les réfugiés contribuent à cet apport. Toutefois, le gouvernement subventionnant les biens et services de base pour maintenir les prix à un certain plafond - que la majorité des réfugiés ne pourrait dépasser -, la contribution de ces derniers au budget de l'État reste inférieure à la charge réelle qu'ils représentent pour le pays.

Leur présence a également entraîné une augmentation de la demande dans le domaine du logement: les Soudanais les accusent volontiers d'avoir provoqué la hausse des loyers. Une étude récente sur la ville de Kassala infirme cependant cette hypothèse : la 
hausse des loyers ne peut être considérée en dehors du phénomène général de l'inflation qui, lui, ne peut être attribué exclusivement aux réfugiés (Kuhlman et al., op. cit.). Cette étude montre également que la hausse des loyers reste proportionnellement inférieure au taux général de l'inflation dans l'ensemble du pays. Alors que les prix des produits de base à Kassala ont accusé une inflation de $1.800 \%$ entre 1980 et 1986, la hausse dès loyers n'a pas dépassé $600 \%$. Néanmoins, la demande de logement étant peu flexible par nature, la hausse des prix dans ce domaine est toujours ressentie de manière aiguë, une part croissante du budget des ménages étant consacrée au loyer. Par contre, la consommation d'autres produits (viande, fruits, etc.) peut être réduite ou même supprimée. Il est aussi intéressant de signaler que l'affluence des réfugiés s'est accompagnée d'une forte expansion dans la construction de logements à Kassala, expansion qui a pu être réalisée grâce au recrutement de réfugiés. Il ne faut pas pour autant oublier les effets négatifs de cette expansion: à Kassala, la densité démographique a augmenté de $19 \%$ dans les zones résidentielles. Les plus touchées par cette surpopulation sont les familles de réfugiés qui, souvent, sont obligées de partager un logement. Les familles soudanaises vivant dans ces quartiers à densité croissante ne sont pas nécessairement des locataires: celles qui étaient propriétaires de leur logement ont, ces derniers temps, divisé leur domicile pour pouvoir s'en réserver une partie et louer le reste. La présence des réfugiés a donc permis à ces familles d'augmenter leurs revenus dans un contexte d'inflation galopante.

Perspectives d'avenir

La majorité des réfugiés ne semble pas envisager de s'installer définitivement au Soudan. Presque tous expriment le désir de rentrer dans leur pays d'origine dès que les conditions seront meilleures, et certaines constatations semblent le confirmer.

Tout d'abord, la plupart de ceux qui se sont réfugiés au Soudan pour fuir la famine de 1984-85 sont effectivement rentrés chez eux lorsque la situation s'est améliorée. En 1986, plus de 600.000 réfugiés du Tigré ont été rapatriés de leur plein gré. Si l'on parvient à un règlement pacifique du problème éthiopien, la majorité des expatriés restant va probablement en (aire autant. Il semblerait que les réfugiés tchadiens regagnent eux aussi leur pays à la suite du changement de régime, mais ils sont rapidement remplacés par d'autres de leurs compatriotes.

Ensuite, certains choix effectués par la majorité des réfugiés semblent indiquer qu'ils ont l'intention de rentrer à terme dans leur pays d'origine : le choix de l'enseignement privé pour leurs enfants, par exemple, est significatif. Dans les établissements privés en effet, dirigés par des réfugiés, des ONG relevant de différents fronts de libération ou des missionnaires, l'enseignement est dispensé dans la langue maternelle de l'enfant, contrairement aux écoles publiques où l'arabe est la langue d'enseignement. Si l'on ajoute à cela le choix de programmes proches de ceux en vigueur dans leur pays d'origine, on peut en déduire qu'ils préparent leurs enfants au retour.

Enfin, les réfugiés tiennent le plus souvent à garder leur identité et ne cherchent pas à être assimilés à la culture ou à la société d'accueil. Ceci ne s'applique évidemment pas à la population érythréenne des Béni Amer ou aux Tchadiens de Zaghawa qui, eux, partagent des caractères socioculturels (langue, religion, appartenance religieuse, etc.) non seulement avec des membres de mêmes tribus de l'autre côté des frontières, mais aussi avec des groupes musulmans voisins au nord du Soudan. Cependant, les Érythréens chrétiens, les Tigréens, les Oromo et les Amhara adoptent un style de vie et des structures normatives différents et semblent soucieux de les conserver (assister à la 
messe à l'église éthiopienne, porter le costume national, etc.). De tels efforts en vue de conserver leur identité confèrent à la communauté des réfugiés les caractéristiques des groupes étrangers dont le séjour est provisoire. Comme le prouve l'expérience de la région d'Equatoria avec les réfugiés ougandais et zaïrois, une petite minorité de réfugiés spontanés pourraient ne pas envisager de rentrer, la situation socioéconomique de leur pays d'origine les en dissuadant.

Le gouvernement soudanais veille à décourager l'installation permanente des réfugiés. La naturalisation de personnes ayant le statut de réfugié est interdite par la loi, indépendamment de la durée du séjour au Soudan. Même pour ceux dont le conjoint est soudanais, il est très difficile d'acquérir la nationalité.

Le fait que les réfugiés expriment le désir de rentrer dès que les causes de leur exil auront disparu ne signifie pas que leur présence au Soudan sera de courte durée. Au début de l'arrivée d'immigrés dans l'est du pays, le gouvernement, la population locale et la communauté internationale ont parié sur la brièveté de leur séjour, et tout a été fait dans ce sens. Or, trois décennies se sont écoulées et le nombre de réfugiés ne cesse de croître. Il en est de même, semble-t-il, des réfugiés tchadiens. Le besoin urgent se fait donc sentir d'une stratégie claire qui, tout en aidant au rapatriement des réfugiés, aurait pour objectif d'encourager ceux qui ne l'ont pas encore fait à se prendre en charge et d'atténuer les effets négatifs de leur présence.

51 Aider les réfugiés à regagner leurs pays respectifs revient à s'efforcer de rétablir la paix dans la région, tâche considérable s'il en est. Plus d'une fois, au cours des dernières années, alors que la paix semblait toute proche dans l'un ou l'autre des pays voisins, un nouveau conflit civil éclatait. Néanmoins, le Soudan et la communauté internationale devraient déployer tous leurs efforts pour rétablir la paix dans la région.

Parallèlement cependant, les programmes pour réfugiés ne devraient pas être conçus à trop court terme. L'étroitesse de vue coûte de plus en plus cher, aussi bien aux réfugiés qu'au pays d'accueil. En particulier, la situation de ceux qui se sont installés par leurs propres moyens demande à être considérée dans le cadre d'une stratégie à plus long terme. Le manque de sécurité et de soutien de cette catégorie tend à aggraver la crise vécue par leurs hôtes. Le concept d'aide aux régions accueillant des réfugiés pourrait constituer un cadre adéquat par lequel transiteraient les fonds destinés à améliorer les conditions de vie des réfugiés et de leurs hôtes. En attendant, tant que le Soudan ne reconnaît pas le statut légal de réfugiés à ceux qui se sont installés par leurs propres moyens, obtenir une aide extérieure lui est difficile.

\section{BIBLIOGRAPHIE}

Al-Shazali S. I., Kuhlman T. et al., Refugees in Rural Development. Final Report of the Research Project "Eritreans in Kassala", Amsterdam, the Free University, 1987.

Al-Shazali S. I., Refugees and Development. The Human Dimension (Conférence sur les dimensions humaines des programmes d'ajustement structurel en Afrique), Khartoum, 1988. 
Al-Shazali S. I., Al-Hassan A. M., The Political Economy of Crises in the Sudan (atelier sur les Érythréens de Kassala), Khartoum University and Free University of Amsterdam, 1987.

National Technical Committee, Report of the National Technical Committee on the Conditions of Refugees in the Sudan, Khartoum, General Secretariat of the Council of Ministers, 1988.

INDEX

Mots-clés : Soudan, réfugiés

\section{AUTEUR}

SALAH AL-DIN AL-SHAZALI

Université de Khartoum 